\title{
TSP50 promotes the Warburg effect and hepatocyte proliferation via regulating PKM2 acetylation
}

Feng Gao ${ }^{1}$, Xiaojun Zhang ${ }^{2}$, Shuyue Wang ${ }^{1}$, Lihua Zheng ${ }^{2}$, Ying Sun ${ }^{3}$, Guannan Wang ${ }^{3}$, Zhenbo Song ${ }^{1}$ and Yongli Bao $\mathbb{D}^{2}$

\begin{abstract}
Metabolic reprogramming is a hallmark of malignancy. Testes-specific protease 50 (TSP50), a newly identified oncogene, has been shown to play an important role in tumorigenesis. However, its role in tumor cell metabolism remains unclear. To investigate this issue, LC-MS/MS was employed to identify TSP50-binding proteins and pyruvate kinase M2 isoform (PKM2), a known key enzyme of aerobic glycolysis, was identified as a novel binding partner of TSP50. Further studies suggested that TSP50 promoted aerobic glycolysis in HCC cells by maintaining low pyruvate kinase activity of the PKM2. Mechanistically, TSP50 promoted the Warburg effect by increasing PKM2 K433 acetylation level and PKM2 acetylation site (K433R) mutation remarkably abrogated the TSP50-induced aerobic glycolysis, cell proliferation in vitro and tumor formation in vivo. Our findings indicate that TSP50-mediated low PKM2 pyruvate kinase activity is an important determinant for Warburg effect in HCC cells and provide a mechanistic link between TSP50 and tumor metabolism.
\end{abstract}

\section{Introduction}

In the presence of sufficient levels of oxygen, normal quiescent cells metabolize glucose to pyruvate, which is further oxidized through oxidative phosphorylation to generate ATP for cellular processes while more glucose is metabolized into lactate under hypoxic conditions. However, even in the presence of sufficient oxygen, tumor cells can reconstruct the process of glucose metabolism, limiting energy metabolism largely to glycolysis, which is called aerobic glycolysis or Warburg effect ${ }^{1,2}$. This cell metabolism reprogramming allows tumor cells to maintain the balance between energy demand and synthetic

\footnotetext{
Correspondence: Zhenbo Song (songzb484@nenu.edu.cn) or

Yongli Bao (baoyl800@nenu.edu.cn)

${ }^{1}$ National Engineering Laboratory for Druggable Gene and Protein Screening, Northeast Normal University, Changchun, China

${ }^{2}$ Research Center of Agriculture and Medicine Gene Engineering of Ministry of Education, Northeast Normal University, Changchun, China

Full list of author information is available at the end of the article

These authors contributed equally: Feng Gao, Xiaojun Zhang

Edited by J.-E. Ricci
}

metabolism, which is conducive to the synthesis of biomacromolecules to promote cell proliferation ${ }^{3}$. The increase of aerobic glycolysis is a widely observed feature in human cancers and often correlates with tumor invasiveness and poor prognosis of human hepatocellular carcinoma (HCC) $)^{4-6}$.

The activation of oncogenes plays an important role in driving the development of tumor metabolism for improving the adaptability of tumor cells and promoting cells proliferation and survival ${ }^{7-11}$. Importantly, interfering with the metabolic changes of tumor cells reduces tumorigenicity and increases apoptosis sensitivity to chemotherapeutic drugs, indicating that aerobic glycolysis metabolism is a key metabolic factor for tumor development ${ }^{12-15}$. Thus, a better understanding of the mechanistic links between cellular metabolism and regulatory genes can be great significance for the development of new treatments, especially in HCC, considering the close correlation between metabolic changes and the pathogenesis of HCC. In the physiological state, TSP50 is

\section{(c) The Author(s) 2021}

(c) (i) Open Access This article is licensed under a Creative Commons Attribution 4.0 International License, which permits use, sharing, adaptation, distribution and reproduction cc) in any medium or format, as long as you give appropriate credit to the original author(s) and the source, provide a link to the Creative Commons license, and indicate if changes were made. The images or other third party material in this article are included in the article's Creative Commons license, unless indicated otherwise in a credit line to the material. If material is not included in the article's Creative Commons license and your intended use is not permitted by statutory regulation or exceeds the permitted use, you will need to obtain permission directly from the copyright holder. To view a copy of this license, visit http://creativecommons.org/licenses/by/4.0/. 
specifically expressed in spermatocytes of testis, while high expression of TSP50 can be detected in more than $90 \%$ of breast cancer, laryngeal cancer, colorectal cancer, cervical cancer and gastric cancer tissue samples ${ }^{16-20}$, suggesting that TSP50 is of great significance in the diagnosis of tumors. After knocking down TSP50 in mouse teratoma cell line P19 and laryngeal cancer cell Hep2, the ability of cell proliferation and colony formation are decreased significantly ${ }^{21}$, and obvious apoptosis occurs ${ }^{17}$. In addition, the natural compounds Alantolactone and Cardamom have been found to significantly reduce the TSP50 level, thereby inhibiting the gastric cancer cells and breast cancer cells proliferation and inducing their apoptosis ${ }^{22}$. We have recently shown that TSP50 can promote CHO cells and MCF-10A cells proliferation, clonal formation and tumorigenic ability and TSP50 knocking-down exerts an inhibitory effect on the migration and invasion of MDA-MB-231 and MDA-MB$435 \mathrm{~S} \mathrm{cells}^{23}$. Meanwhile, the function of TSP50 in promoting invasion and metastasis is mainly achieved by increasing the expression of NF-kB-dependent $\mathrm{MM}^{23}$. Further studies have shown that ІкB $\alpha$, a key signal molecule of NF-kB signal pathway, is degraded after binding to TSP50, thus promoting cell proliferation and inducing cell transformation ${ }^{24}$. The threonine protease activity and catalytic triad of TSP50 are essential for its function in cell proliferation ${ }^{25,26}$. In breast cancer cells, TSP50 can also promote cell proliferation partly by inhibiting activin signal transduction ${ }^{16}$. These findings suggest that TSP50 plays an important role in the diagnosis, prognosis and treatment of tumors. However, whether the proliferation-promoting function of TSP50 is partly due to the increased Warburg effect in human cancer cells has not been elucidated before.

PKM2 is recognized as the key rate-limiting enzyme that regulates aerobic glycolysis in tumor cells, catalyzing the synthesis of pyruvate and ATP using phosphoenolpyruvate and $\mathrm{ADP}^{27,28}$. PKM1 and PKM2 are encoded by the PKM gene. PKM1 is mainly expressed in muscle or brain tissues that need a large amount of energy supply, whereas PKM2 is significantly expressed in rapidly proliferating tissues such as embryonic cells, stem cells or tumor cells ${ }^{29,30}$. PKM2 in tumor cells, but not its spliced variant PKM1, is often depolymerized into low-activity dimers or inactive monomers, resulting in a decrease of PK activity that promotes the Warburg effect and favors cancer cell growth and survival ${ }^{31-33}$. On the contrary, the high PK activity of PKM2 exerts an inhibitory effect ${ }^{33-35}$, emphasizing the importance of identifying endogenous regulators of PKM2 activity in cancer.

In this study, we found that TSP50 was highly expressed in a variety of HCC cells. TSP50 could directly bind to PKM2 to positively regulate aerobic glycolysis through the influence of PKM2 pyruvate kinase activity, thus promoting the survival of HCC cells. We demonstrated that TSP50 maintained the low pyruvate kinase activity of PKM2 by directly mediating the acetylation of PKM2 at K433. In summary, we reveal a molecular link between cell survival regulators and key enzymes of cancer metabolism and our study will provide potential therapeutic significance for HCC patients.

\section{Results}

TSP50 is highly expressed in HCC and survival-associated

We evaluated TSP50 transcription levels via UALCAN database ${ }^{36}$. Analysis results revealed that mRNA expression of TSP50 was significantly higher in TCGA-LIHC tissues than in adjacent normal tissues (Fig. 1A). Further sub-group analysis of multiple clinic-pathological features of TCGA-LIHC samples based on disease stages, gender, age, and tumor grade showed that the expression of TSP50 was significantly higher in HCC patients than normal controls (Fig. 1B-E). The comparisons results made within different tumor stages and grades showed that the expression level of TSP50 in tumor grade 2 subgroup was significantly lower than that in grade 3 group, however, there was no significant difference among other subgroups, suggesting that TSP50 expression was not stage or grade dependent (Fig. 1B and E). Then, GEPIA was employed to generate survival curves (recurrence-free survival,RFS) to assess the association between TSP50 expression and the survival outcomes of HCC cohorts ${ }^{37}$. The HCC patients were separated into two groups according to the TSP50 mRNA expression level. Finally, we found that the high TSP50 expression group had a shorter RFS (Fig. 1F). Thus, TSP50 expression may serve as a potential diagnostic indicator in $\mathrm{HCC}$.

\section{TSP50 promotes cell proliferation and drives glycolytic metabolism in HCC cells}

The expression of TSP50 in different cell lines (L02 cells, SMMC-7721 cells, Huh7 cells, HepG2 cells and Bel7402 cells) was detected. Western blot results showed that TSP50 was almost not detected in normal L02 cells, whereas higher expressed TSP50 was found in Huh7 and Bel7402 HCC cells (Fig. 2A). To investigate the effect of TSP50 on cell proliferation, Huh7 and Bel7402 cells were transfected with shTSP50 plasmids for TSP50 knockdown (Fig. 2B, C). MTT and BrdU results showed that TSP50 knockdown significantly inhibited Huh7 and Bel7402 cell viability and proliferation (Fig. 2D-E, N-O). Studies have indicated that tumor cells underwent metabolic reprogramming to adapt to rapid proliferation and enhanced the aerobic glycolysis pathway ${ }^{38}$. To figure out whether alteration of TSP50 directly influence glycolytic metabolism, we measured extracellular acidification rate (ECAR), oxygen consumption rate (OCR), glucose consumption, lactate production, LDH activity, ATP, G6P and 2-PG 

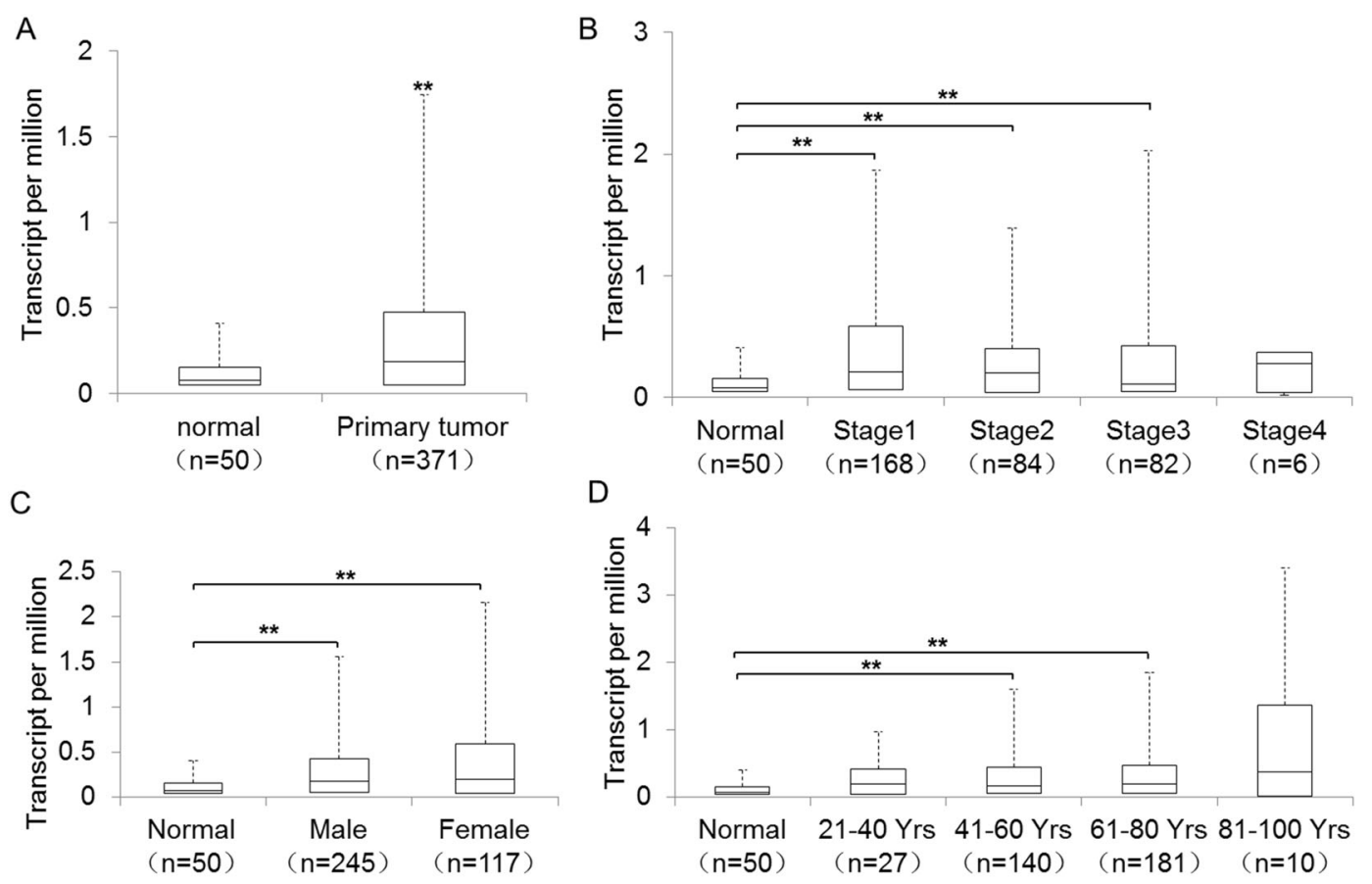

D
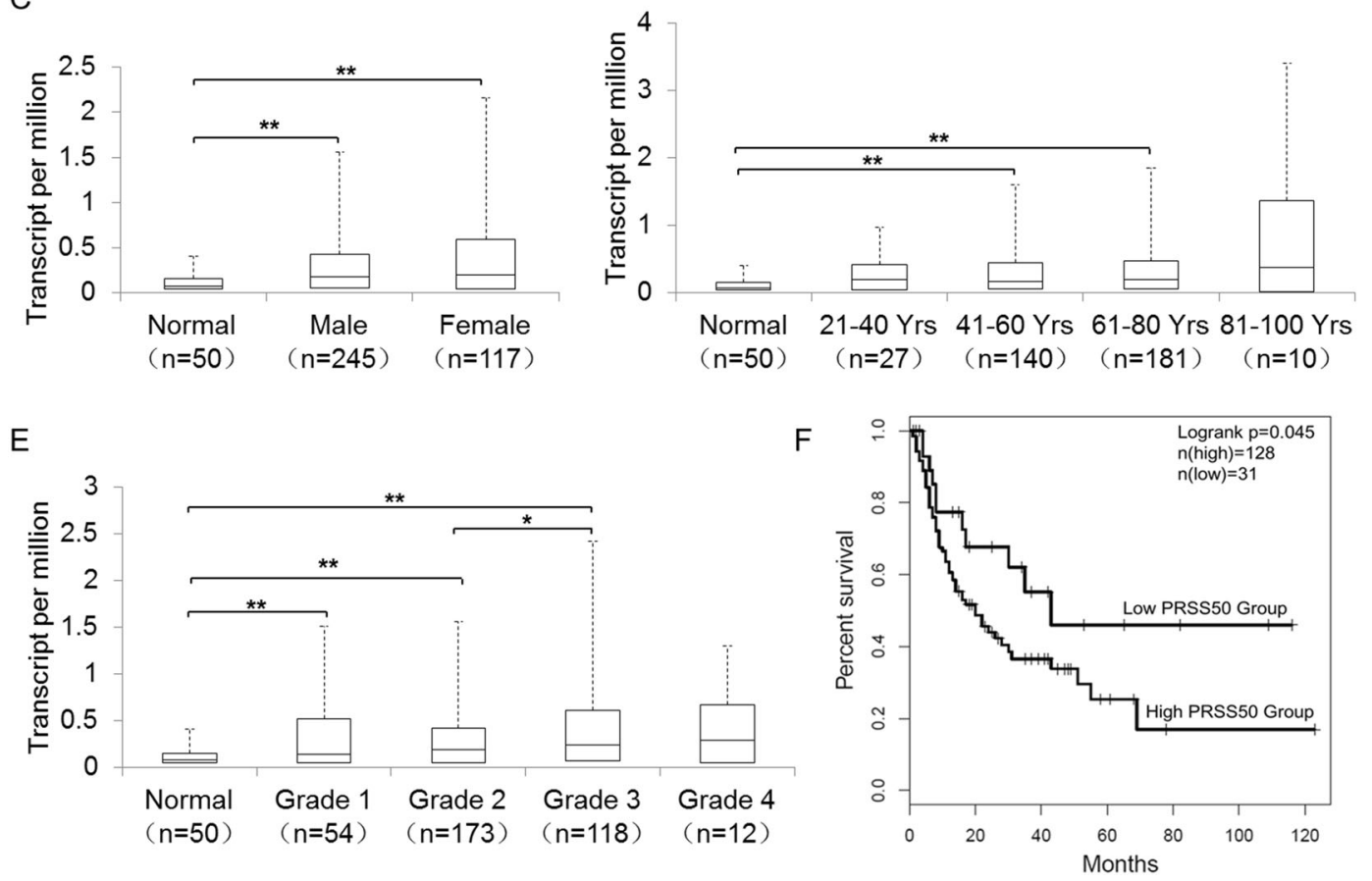

Fig. 1 TSP50 is elevated in HCC and survival-associated. A Boxplot showing relative expression of TSP50 in normal and HCC samples. B Boxplot showing the relative expression of TSP50 in normal individuals and HCC patients in different stages. C Boxplot showing the TSP50 relative expression in normal individuals as well as male and female HCC patients, respectively. D Boxplot showing relative expression of TSP50 in normal individuals of any age and LIHC patients aged 21-40, 41-60, 61-80, or 81-100 yr. E Boxplot showing the TSP50 relative expression in normal individuals and HCC patients with grade 1, 2, 3 or 4 tumors. F GEPIA analysis of the correlation between TSP50 mRNA expression levels and RFS of HCC patients. A-E UALCAN database. F GEPIA database. The Student's $t$-test was used to estimate the significance of difference between two groups and more than two groups were analyzed by one-way ANOVA. ${ }^{*} P<0.05,{ }^{*} P<0.01$.

levels in HCC cells after TSP50 knockdown. The results showed that TSP50-knockdown HCC cells revealed lower ECAR levels and higher OCR levels, meanwhile, other aerobic glycolysis related indicators levels were also significantly reduced (Supplementary Fig. S1, Fig. 2F-M, $\mathrm{P}-\mathrm{W})$. Subsequently, we overexpressed TSP50 with pcDNA3.1-TSP50, which induced the overproliferation of L02 cells (Fig. 3A-C). Further functional colorimetric validation showed that TSP50 increased glucose consumption, lactate production, LDH activity, ATP as well as G6P and 2PG levels (Fig. 3D-I). Meanwhile, TSP50 overexpression increased ECAR levels and reduced OCR levels in L02 cells compared with control cells (Supplementary Fig. S1, Fig. 3J, K). After obtaining the above results, we further examined the effect of TSP50 on the levels of regulatory factors in the glycolytic pathway. We found that TSP50 could promote the expression of glycolysis-related proteins such as GLUT1, HK2, PKM2, etc (Supplementary Fig. S2).

In addition, 2-deoxy-d-glucose (2-DG) was applied to TSP50-overexpressing L02 cells to block glycolysis and we observed that the levels of aerobic glycolysis and the proliferation ability of L02 cells were significantly decreased. In detail, the ability of aerobic glycolysis 


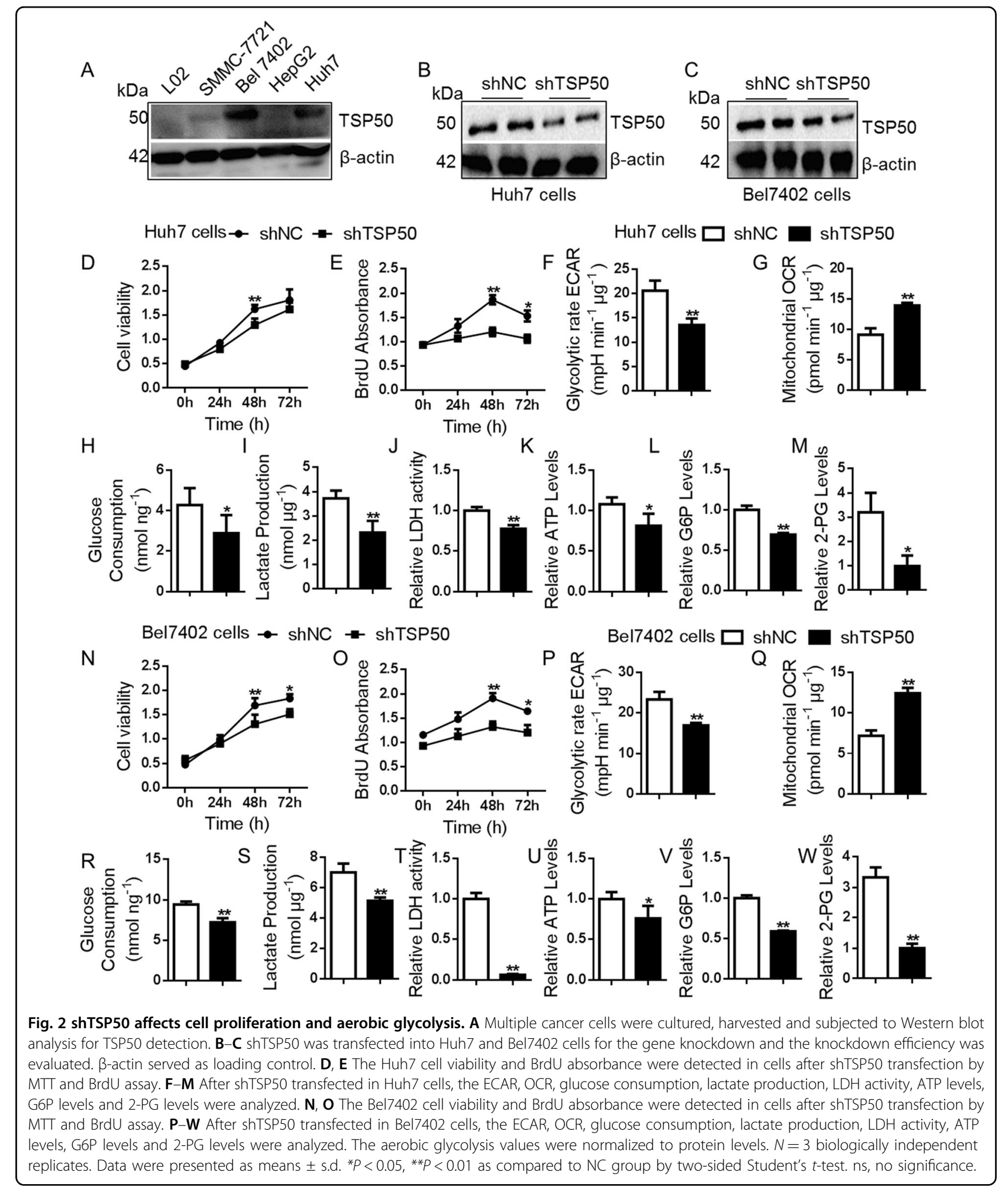

and cell proliferation in TSP50 + 2-DG group were significantly higher than 2-DG group and lower than those in the TSP50 overexpression group (Supplementary Fig. S3, Fig. 3L-R). Taken together, our data suggested that TSP50 was beneficial to cell proliferation and aerobic glycolysis, and the tumor-promoting effect of TSP50 was partly dependent on aerobic glycolysis. 


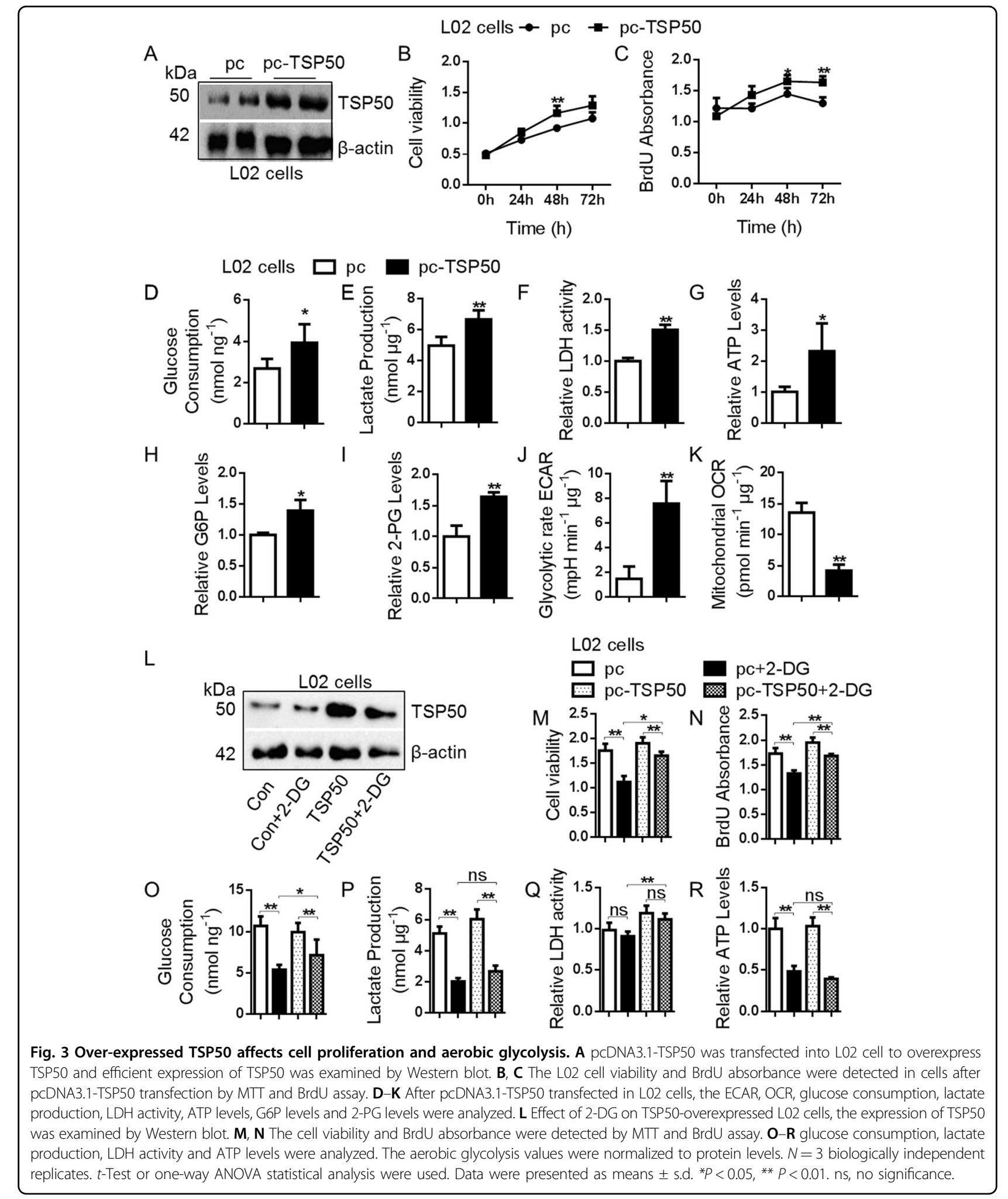

TSP50 interacts with PKM2 and depends on PKM2 for its metabolic effects

As TSP50 could apparently promote aerobic glycolysis, we infer that TSP50 may bind to metabolic enzymes, thereby regulating aerobic glycolysis. To identify the underlying target molecules regulated by TSP50, LC-MS/MS was performed and the results showed that PKM was one of the proteins interacting with TSP50 (Fig. 4A, data not shown). 
A
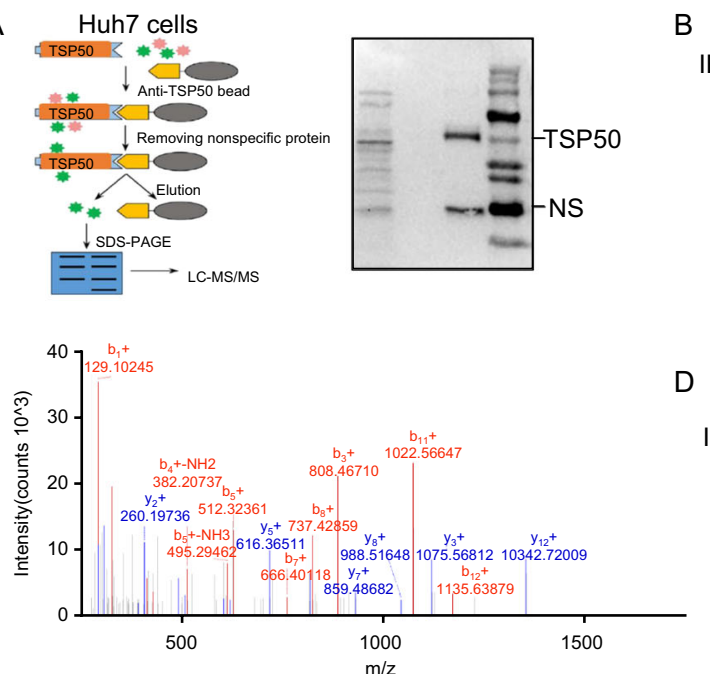

B

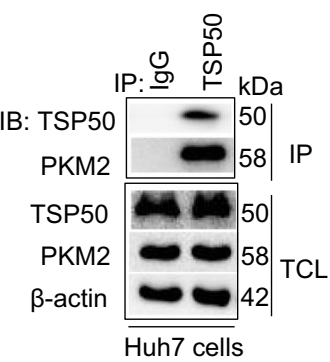

$D$

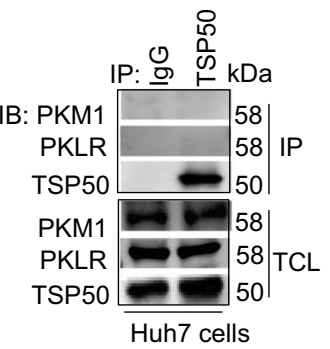

C IP: 垈 $\frac{\stackrel{0}{0}}{5}$

IB: TSP50

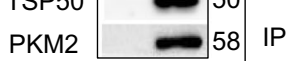

TSP50

PKM2

$\beta$-actin

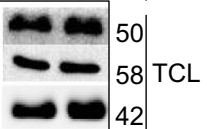

Bel7402 cells

E

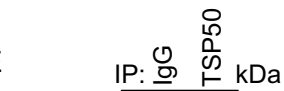

IB: PKM1

PKLR $\square$ IP

TSP50 -50

PKM1 58

PKLR $\longrightarrow 58{ }_{\mathrm{TCL}}$

TSP50 50

Bel 7402 cells
F GST $\frac{\text { Huh7 cells }}{++-\quad-}$

GST-TSP50 - - + +

Flag-PKM2 $++++\mathrm{IB}$ :

$\mid \begin{array}{lll}\mathrm{kDa} & & \\ 78 & & \end{array}$

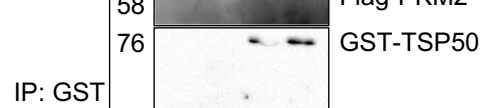

$26 \multimap$ GST

$58 \longrightarrow-2$ Flag-PKM2

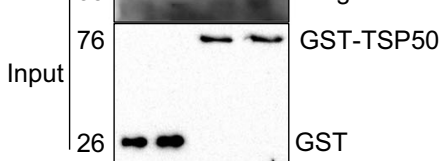

G

Bel7402 cells

GST-TSP50 - - + +

Flag-PKM2 $++++\mathrm{IB}$ :
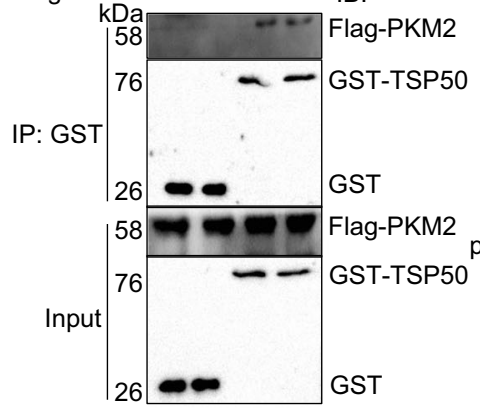

$\mathrm{H}$
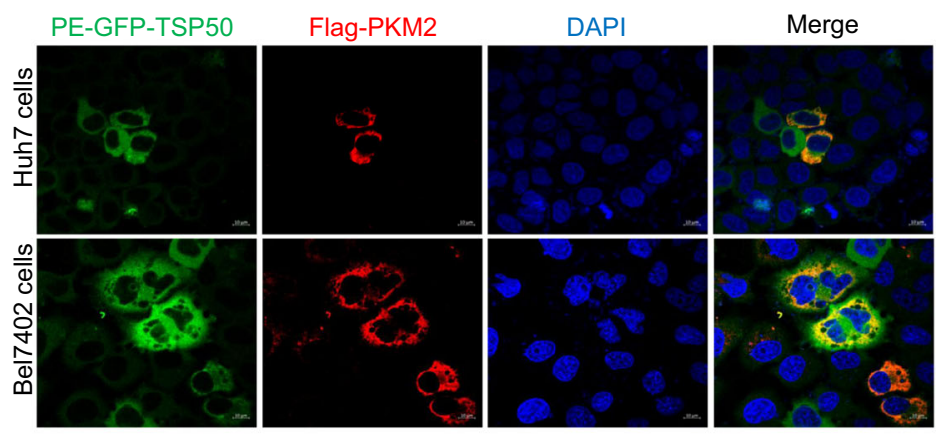

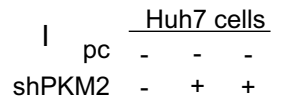

ShPKM2 - +

kDa: $58 \longrightarrow$ PKM2
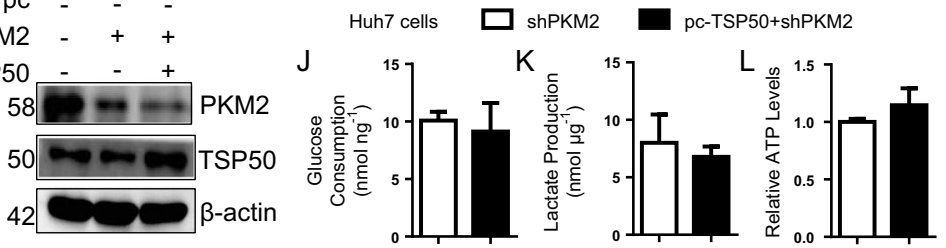
Bel7402 cells

ShPKM2 ++-

pc-TSP50
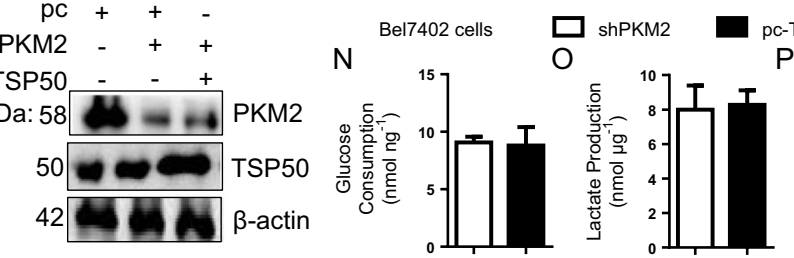

PC-TSP50+shPKM2

$P$

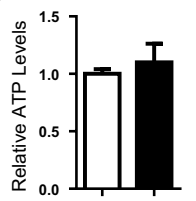

Fig. 4 TSP50 binds with PKM2 and depends on PKM2 for its metabolic effects. A Identification of proteins binding to endogenous TSP50 by LCMS/MS in Huh7 cells. Candidate binding proteins to TSP50 were obtained after non-specific proteins were removed. B, C Huh7 and Bel7402 cells were harvested and subjected to immunoprecipitation with anti-TSP50 antibody, followed by Western blot analysis with anti-PKM2 antibody. D, E Huh7 and Bel7402 cells were harvested and subjected to immunoprecipitation with anti-TSP50 antibody, followed by Western blot analysis with antiPKM1 or anti-PKLR antibody. F, G GST pull-down of Flag-PKM2 by GST-TSP50 using proteins purified in B21 bacteria, followed by Western blot analysis with anti-PKM2 and anti-GST antibody. H Huh7 and Bel7402 cells were fixed and subjected to immunofluorescence analysis. TSP50 co-localized with PKM2 in Huh7 and Bel7402 cells. Scale bar, 10 m. I-P Glucose consumption, lactate production and ATP levels were analyzed after silencing of PKM2 abrogates the metabolic effects of TSP50. All aerobic glycolysis values were normalized to protein levels. $N=3$ biologically independent replicates. $t$ Test statistical analysis was used. Data were presented as means \pm s.d. 


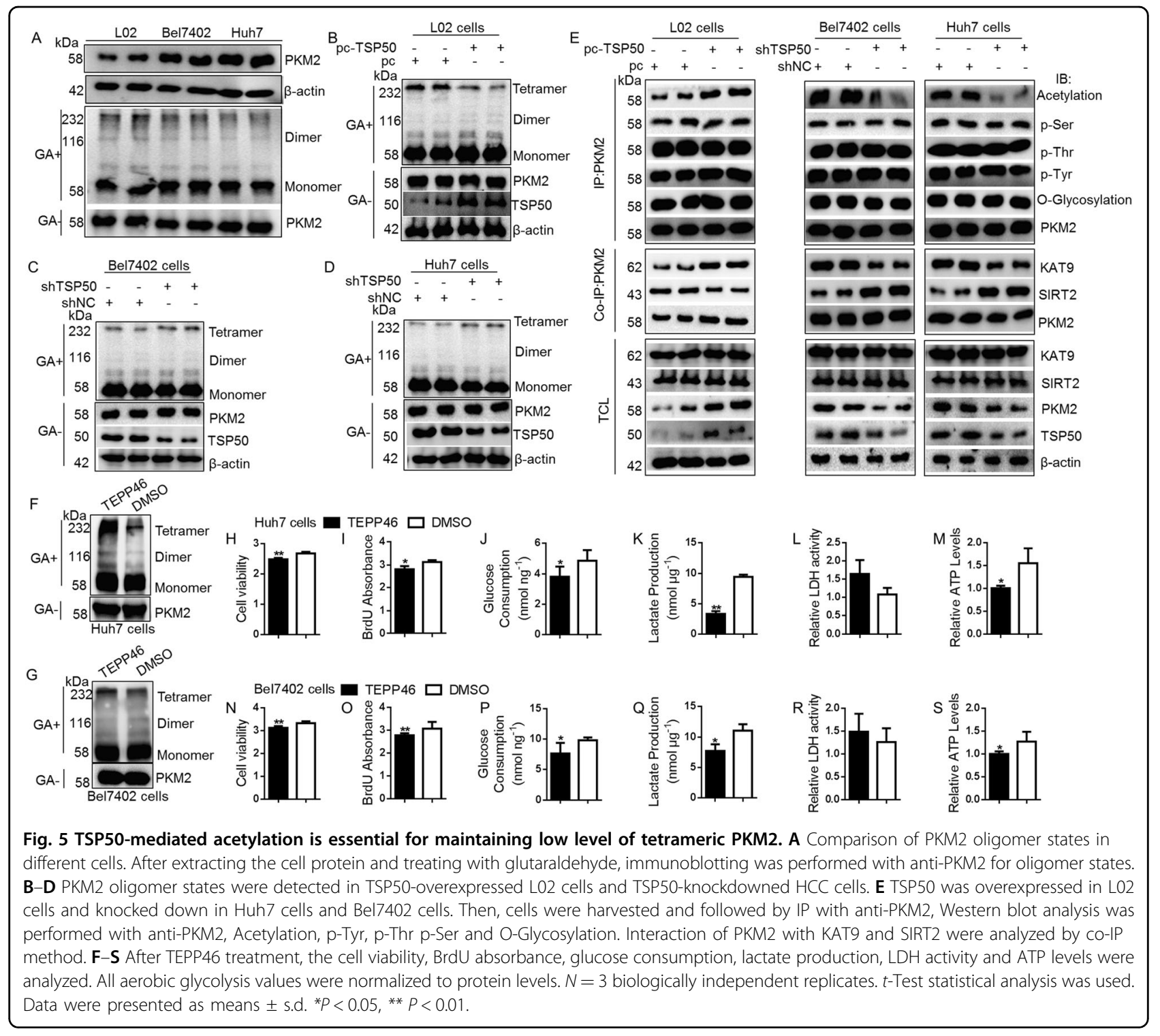

We verified this result with co-IP assay, which showed that endogenous TSP50 protein interacted with PKM2 protein in the cytoplasm rather than PKM1 or PKL/R (Fig. 4B-E). Recent studies have shown that PKM2 is a major regulator for metabolic reprogramming in cancer which contributes to the Warburg effect ${ }^{28,39,40}$. To further validate the interaction between TSP50 and PKM2 in HCC cells, GST pulldown and immunofluorescence assays were performed. We found that GST-tagged TSP50 protein could pull-down Flag-tagged PKM2 protein by Western blotting upon GST pull-down assays (Fig. 4F, G). As confirmed by Immunofluorescence, TSP50 and PKM2 were co-localized in the cytoplasm after GFP-TSP50 and Flag-PKM2 plasmids were co-transfected into Huh7 and Bel7402 cells (Fig. 4H). All the results above indicated that there was a close direct interaction between TSP50 and PKM2 proteins.
Further results showed that the effect of TSP50 on aerobic glycolysis level was significantly reduced in PKM2-knockdown Huh7 and Bel7402 cells (Fig. 4I-P), which confirmed that TSP50 was dependent on PKM2 for its metabolic effects.

\section{TSP50-mediated acetylation is essential for maintaining low level of tetrameric PKM2}

In cancer cells, a constitutively low PKM2 activity is essential for aerobic glycolysis ${ }^{31-33}$. The oligomers of PKM2 exist in high activity tetramer, low activity dimer and inactive monomer forms. Therefore, we investigated a possible relationship between PKM2 and TSP50. We first compared the oligomeric state of PKM2 in different cells, and the results showed that, compared with L02 cells, the high-activity tetrameric form in Huh7 cells and Bel7402 
A

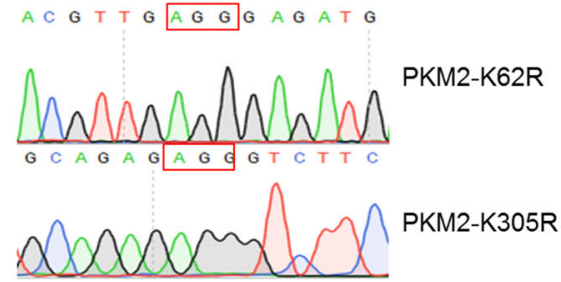

B
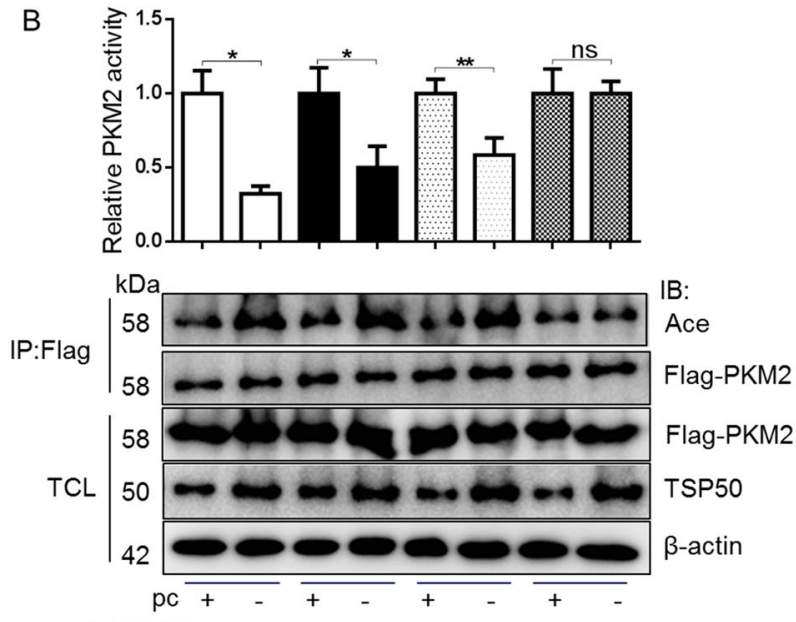
pc1-TSP50

Flag-PKM2-WT + Flag-PKM2-K62R Flag-PKM2-K305R -

Flag-PKM2-K433R -

$\mathrm{F}$

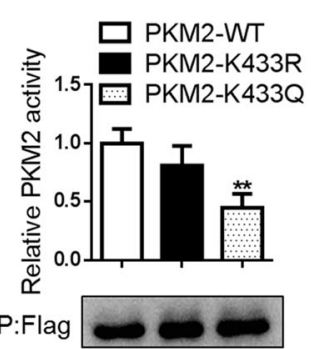

G

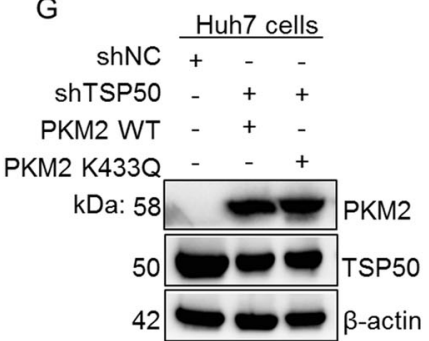

CTCACCAGGTCTGGC
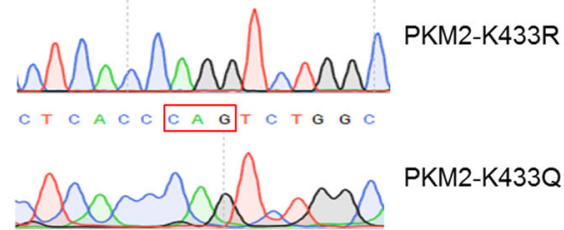

PKM2-K433Q

C

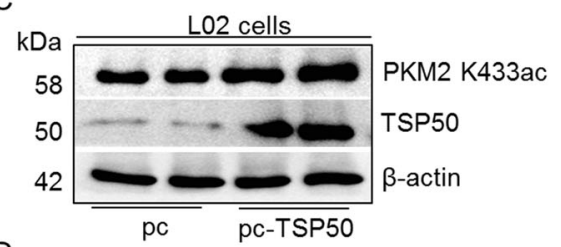

$\mathrm{D}$

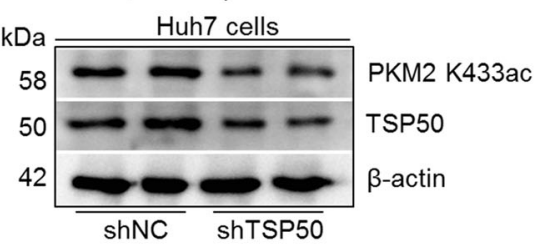

$E_{k}$
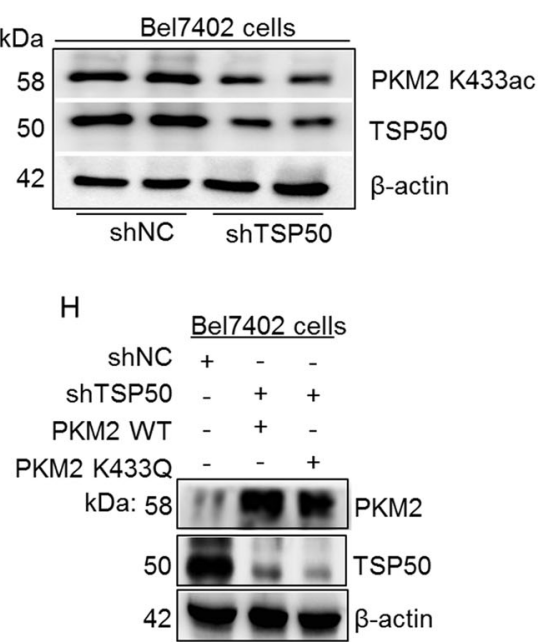

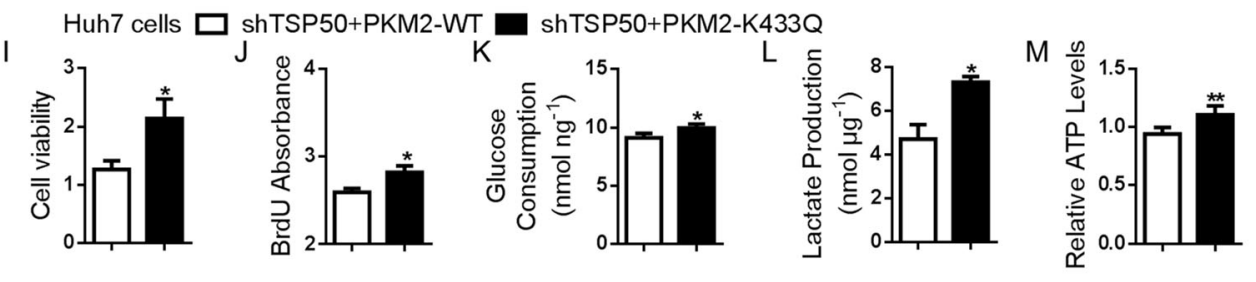

Bel7402 cells $\square$ shTSP50+PKM2-WT $\square$ shTSP50+PKM2 K433Q

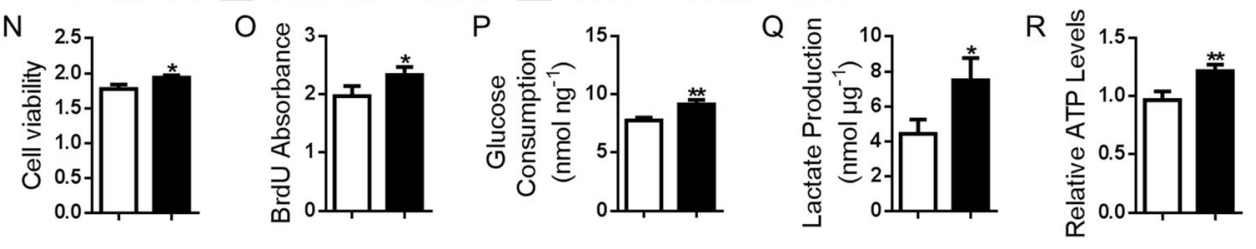

Fig. 6 (See legend on next page.) 
(see figure on previous page)

Fig. 6 TSP50 acetylated PKM2 at K433 site. A Vectors encoding for multiple mutant PKM2 were constructed for subsequent analysis using a sitedirected mutagenesis kit. B PKM2 K62R, K305R or K433R mutant vector was co-transfected with TSP50 into Huh7 cells. The cell was harvested and followed by IP with anti-Flag and PKM2 activity analysis. C-E The pcDNA3.1-TSP50 and Flag-PKM2 WT, Flag-PKM2K62R, Flag-PKM2 K305R or FlagPKM2 K433R mutant plasmids were co-transfected into cells, respectively. The level of PKM2 K433 acetylation was detected using an anti-PKM2 K433ac antibody. F-R Acetylated mimic of PKM2 (PKM2-K433Q) transfected in cell lines deficient of TSP50, the PKM2 activity, cell viability, BrdU absorbance, glucose consumption, lactate production and ATP levels were detected. All aerobic glycolysis values were normalized to protein levels. $N=3$ biologically independent replicates. $t$-Test statistical analysis was used. Data were presented as means $\pm s . d$. ${ }^{*} P<0.05$, ${ }^{*} P<0.01$. ns, no significance.

cells was reduced (Fig. 5A). Furthermore, TSP50 overexpression in L02 cells led to a low level of PKM2 tetrameric form, while TSP50 knockdown in Huh7 and Bel7402 cells significantly increased tetrameric PKM2 (Fig. 5B-D).

The activity of PKM2 is regulated by various covalent modifications, such as phosphorylation ${ }^{41,42}$, acetylation $^{43,44}$ and glycosylation ${ }^{45}$. We used immunoprecipitation assay to examine the effect of TSP50 on PKM2 protein modification. The results showed that TSP50 promoted the acetylation level of PKM2, with negligible effects on phosphorylation and glycosylation (Fig. 5E). Since TSP50 only has the activity of threonine hydrolase, we speculate that TSP50 may affect the binding ability of PKM2 to acetylase or deacetylase. Further detection results showed that TSP50 could reduce deacetylase SIRT2 level and increase acetylase KAT9 level, two proteins that interacted with PKM2 (Fig. 5E). Furthermore, TEPP-46, a PKM2 activator, was selected to detect the effect of altered PKM2 activity on cell proliferation and aerobic glycolysis levels. The results showed that TEPP 46 effectively activated PKM2 in HUH7 and BEL7402 cells (Fig. 5F-G). The proliferation and aerobic glycolysis levels of tumor cells treated with TEPP 46 were significantly lower than the control group cells (Fig. 5H-S). These results indicated that TSP50 inhibited PKM2 pyruvate kinase activity by mediating acetylation of PKM2 thereby promoting aerobic glycolysis and cell proliferation levels.

\section{PKM2 is acetylated at $\mathrm{K} 433$}

We next sought to identify the PKM2 acetylation site(s) affected by TSP50. The acetylation sites of PKM2 are usually K62 lysine, K305 lysine and K433 lysine ${ }^{44,46,47}$. Therefore, the mutant plasmids of Flag-PKM2-K62R, K305R and K433R were constructed and transfected into Huh7 cells (Fig. 6A). Subsequently, after transfecting TSP50 in PKM2-WT, K62R, K305R and K433R cells, different forms of PKM2 protein were purified by immunoprecipitation, and the acetylation levels were detected. The results showed that PKM2 acetylation levels and PKM2 pyruvate kinase activity in PKM2 K433R cells were not affected by TSP50 (Fig. 6B). Finally, Western blot detection results with anti-PKM2 K433ac antibody showed that TSP50 could promote the acetylation level of
PKM2 K433 site (Fig. 6C-E). Together, these results demonstrated that TSP50 acetylated PKM2 at K433.

\section{TSP50 mediates the cell proliferation and aerobic glycolysis changes partially dependent on PKM2 K433 acetylation}

Studies have shown that pyruvate kinase mutations affecting PKM2 activity and thus cancer metabolism ${ }^{48}$. The PKM2 pyruvate kinase activity is significantly lower when PKM2 lysine 305 is substituted with glutamine $(\mathrm{K} 305 \mathrm{Q})^{35,44,46}$. In contrast, PKM2 K62Q mutation has no effect on PKM2 pyruvate kinase activity ${ }^{44,46}$. The effect of PKM2 K433Q on PKM2 pyruvate kinase activity has not been studied, however, it has been shown that the increase of PKM2 K433 acetylation level can reduce the accumulation of PKM2 tetramer, leading to a decreased PKM2 activity $^{49,50}$. In this study, to determine how PKM2 K433 acetylation affects PKM2 activity, we constructed a FlagPKM2-K433Q mutant plasmid (Fig. 6A). The PKM2 WT and PKM2 K433Q mutants were immunopurified from the transfected HEK-293T cells and PKM2 activity was measured. We found that the PKM2 pyruvate kinase activity of PKM2 K433Q mutant was significantly reduced (Fig. 6F). Meanwhile, in TSP50-knockdown Huh7 and Bel7402 cells, when PKM2 lysine 433 was substituted with glutamine $(\mathrm{K} 433 \mathrm{Q})$, cell proliferation and aerobic glycolysis level were increased significantly, as compared to PKM2 WT-transfected control cells (Fig. 6G-R). Together, these results demonstrated a strong effect of high K433 acetylation level in inhibiting PKM2 pyruvate kinase activity and promoting aerobic glycolysis.

Subsequently, the role of PKM2 K433 acetylation in TSP50-induced cell proliferation and aerobic glycolysis was verified. We co-transfected TSP50 with PKM2 K433R into L02 and HCC cells, and the TSP50 + PKM2 WT and TSP50 + PKM2 K62R were used as controls (Fig. 7A). After transfection for $48 \mathrm{~h}, \mathrm{MTT}$ and BrdU results showed that the viability and proliferation level of cells in the K433R group were significantly lower than that in the PKM2 WT and PKM2 K62R groups (Fig. 7B, C, J, K, R-S). The levels of aerobic glycolysis were further detected, and the results showed that glucose consumption, lactate production, ATP, G6P and 2PG levels decreased significantly in K433R group (Fig. 7D-H, L-P, T-X). 


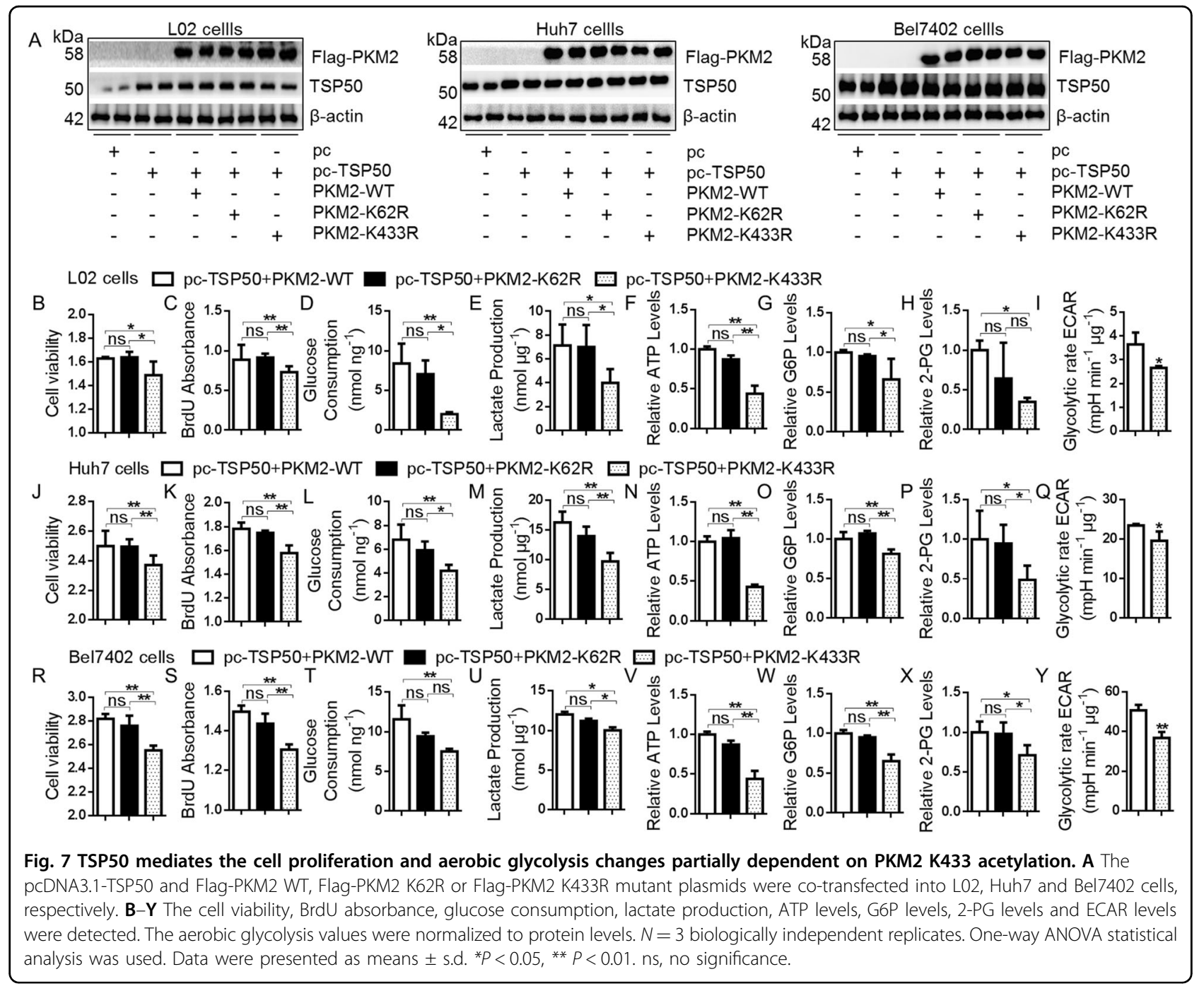

Furthermore, the ECAR levels in the TSP50 and PKM2 K433R expressing group were significantly lower than that in the control groups (Fig. 7I, Q and Y). Taken together, these results suggested that the regulation of TSP50 on cell proliferation through the glycolysis pathway depended on the acetylation level of PKM2 K433 site.

\section{PKM2 K433 acetylation is required for TSP50-induced tumor growth}

To extend our studies into an animal model, we examined the role of PKM2 K433 acetylation in regulating the growth of TSP50-induced tumors in immunedeficient mice in vivo and assayed for tumor formation ( $n=6$ /group). The TSP50 and PKM2 WT-stableexpressed L02 cells were prepared by lentivirus infection (Fig. 8A, B) which were used for female athymic nude mice dorsal subcutaneous injection. All immune-deficient mice in PKM2 WT and TSP50 + PKM2 WT groups emonstrated tumor formation by 4 weeks post-injection.
Compared with the PKM2 WT group, the tumor volume and weight in TSP50+ PKM2 WT group were significantly increased (Fig. 8C-E). The relative expression of TSP50 and PKM2 was consistent with that in stably transfected cells (Fig. 8F, G). Meanwhile, higher lactate levels and lower ATP levels were also detected in TSP50 + PKM2 WT group (Fig. 8H, I).

Furthermore, we constructed a PKM2 K433R-stable expressed L02 cell line for nude mice subcutaneously injection (Fig. 8J, K). After 4 weeks in vivo tumour growth, the mice were sacrificed, and tumour growth was assessed. Compared with the NC group, TSP50 significantly promoted tumor formation. On the other hand, in TSP50overexpressing cells, PKM2 K433R transfection group demonstrated a smaller tumor size (vs. PKM2 WT group as a control), accompanied by a dramatically reduced tumor weight (Fig. 8L-N). The relative expression of TSP50 and PKM2 was consistent with that in stably transfected cells (Fig. 8O, P). In addition, tumor tissues in 


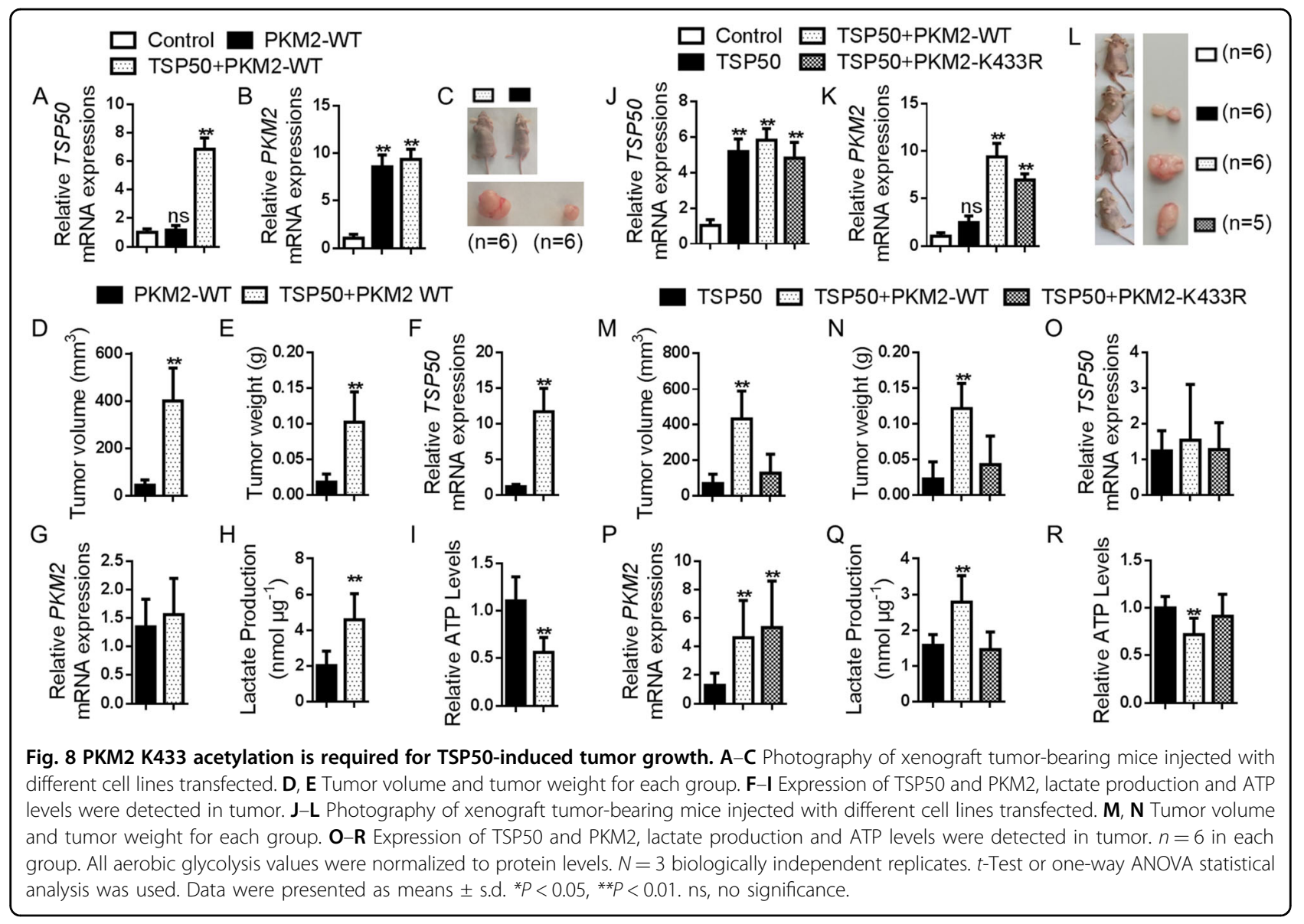

the TSP50 + PKM2 WT group showed increased lactate production and reduced ATP levels (Fig. 8Q-R).

In summary, the results showed that TSP50 obviously enhanced PKM2 WT-cells growth, however, it has no effect on the PKM2 K433R-cells. These findings indicated that TSP50 played a key role in cell proliferation and tumor growth, at least in part, by regulating PKM2 K433 acetylation through aerobic glycolysis pathway.

\section{Discussion}

Metabolic reprogramming is recognized as one of the hallmarks of cancer ${ }^{51}$. Even with sufficient oxygen, the energy is supplied mainly through glycolytic metabolic pathways in tumor cells, which is known as Warburg effect. Aerobic glycolysis signature enables tumor cells to satisfy anabolic and energetic needs for biological macromolecules production. This metabolic is involved in regulating the rapid proliferation and apoptosis resistance of tumor cells ${ }^{38,52}$. Many human cancers, including HCC, exhibit an aerobic glycolytic phenotype, which is often associated with tumor progression and poor clinical outcomes in cancer patients ${ }^{4-6,53,54}$. Therefore, tumorspecific aerobic glycolysis may be potential therapeutic target for HCC treatment. The pyruvate kinase M2
(PKM2) isoform, which is commonly upregulated in many human cancers, has been recently shown to play a crucial role in catalyzing aerobic glycolysis. The decrease of PKM2 activity significantly promotes the level of aerobic glycolysis ${ }^{33,55}$. High glycolysis flux is affected by the activation of oncogenes and mutations of tumor suppressor genes ${ }^{52}$. Nevertheless, the molecular regulatory mechanisms remain unclear.

Here, we demonstrate that oncogene TSP50 promotes the Warburg effect in HCC and reveal an underlying molecular mechanism: TSP50 maintains low PKM2 pyruvate kinase activity by increasing the acetylation level of PKM2 in cytoplasm. Through this regulation, TSP50 can promote the proliferation of HCC cells both in vivo and in vitro and may be a potential molecular target for HCC therapy.

TSP50 is abnormally highly expressed in various tumor tissues, but is absent in normal tissues ${ }^{16-20}$, suggesting a pathogenic role for TSP50 in cancers. TSP50 levels appear to have important clinical implications for cancer patients $^{18,23,56}$, therefore, we evaluated TSP50 transcription levels via UALCAN database. Analysis results revealed that mRNA expression of TSP50 was significantly higher in TCGA-LIHC tissues than in adjacent 
normal tissues. GEPIA database analysis results showed that the high TSP50 expression group had a shorter RFS. Thus, TSP50 expression may serve as a potential diagnostic indicator in HCC and deserves further experimental validation.

In this study, an abnormally high expression of TSP50 was detected in HCC cells and TSP50 level was positively correlated with expression of glycolytic genes, supporting its relevance for the glycolytic phenotype of HCC cells. We further examined the regulation of TSP50 as a protooncogene on the Warburg effect, and we demonstrated that TSP50 knockdown impaired the aerobic glycolytic phenotype and survival in cultured Huh7 and Bel7402 cells. On the contrary, TSP50-overexpressed L02 cells showed an enhanced Warburg effect, which was reversed by a glycolysis inhibitor 2-DG. Meanwhile, the proliferationpromoting effect of TSP50 was partially attenuated by the addition of 2-DG. This suggested that TSP50 may promote both HCC initiation and maintenance at least in part by upregulating glycolytic metabolism.

We speculated that the effect of TSP50 on aerobic glycolysis may be mediated by some key factors such as Glut1, HK2 and PKM2. LC-MS/MS detection results showed that TSP50 might interact with PKM2, and we verified this results. Furthermore, TSP50 is dependent on PKM2 for its metabolic effects. Recent studies have confirmed that the low PK activity of PKM2 plays a key role in promoting the Warburg effect and tumor cell survival ${ }^{31-33}$. We indeed found that TSP50 maintained low PKM2 pyruvate kinase activity in TSP50-transfected L02 cells and this was also demonstrated by the enhanced pyruvate kinase activity of PKM2 in TSP50 knock-downed HCC cells. Studies have shown that PKM2 activity is regulated by metabolic intermediates $^{34}$, phosphorylation modification ${ }^{41,42,57,58}$, acetylation modification ${ }^{43,44}$, O-GlcNAcylation modificatio ${ }^{45}$, protein-protein interaction ${ }^{59,60}$, transcriptional regulation and selective splicing ${ }^{61}$, and miRNAs ${ }^{62,63}$. So how does TSP50 decrease PKM2 pyruvate kinase activity? Our results showed that TSP50 promoted PKM2 K433 acetylation by increasing PKM2-bound KAT9 level, and decreasing SIRT2 level in the complex in cytoplasm. However, we cannot define whether TSP50 can directly acetylate PKM2 which needs further studied. Furthermore, PKM2 K433R mutation inhibited TSP50-induced tumor formation and aerobic glycolysis in immune-deficient mice in vivo.

It is known that high acetylation level of PKM2 can reduce its enzyme activity and promote aerobic glycolysis ${ }^{43,44}$, which is consistent with our findings in HCC cells. Studies on PKM2 K433 acetylation show that PKM2 is acetylated at $\mathrm{K} 433$ by p300 and function as a protein kinase for gene expression regulation in nucleus to promote cell proliferation ${ }^{43}$. A previous study also showed that the MCL treatment reduces the acetylation at K433, contributing to the tetramerization of PKM2 indirectly and inhibiting nuclear translocation in cells, which further suppressing the transcriptional regulation in tumorigenesis $^{50}$. In the activated dendritic cells (DCs) induced by LPS, JNK promotes PKM2-K433ac, resulting in the decreased PKM2 enzymatic activity and high glycolysis level ${ }^{49}$. PKM2 also have many nonglycolytic functions. For example, PKM2 K433 acetylation is found to facilitate PKM2-DDB2 binding, thus affecting cell survival, which is consistent with UV irradiation ${ }^{64}$. However, in our study, we mainly analyzed the effect of TSP50-mediated PKM2 K433 acetylation on its pyruvate kinase activity in the cytoplasm. We found that PKM2 was acetylated at K433 by KAT 9 and deacetylated by SIRT2. Interestingly, studies have shown that PKM2 lysines 305 is a direct SIRT2 deacetylation targets ${ }^{46}$. Recent studies have also suggested HDAC7 to deacetylate PKM2 at lysine $433^{65}$. We speculate that this may be regulated by TSP50. However, these inferences need further analysis to be confirmed. Additionally, we cannot clarify the role of TSP50 in affecting the binding ability of PKM2 to KAT9 or SIRT2 and the exact regulatory mechanism is unclear. Therefore, it is necessary to conduct further experiments to analyze this phenomenon. Furthermore, TSP50 may regulate tumor metabolism through a variety of pathways. Are these pathways acting independently or intersecting? Which is most effective? These problems also need to be further investigated.

In conclusion, TSP50 acetylates the PKM2 K433 site for low PKM2 pyruvate kinase activity to promote the Warburg effect, which is benefit for HCC cells survival, and we reveal a new regulatory mechanism of TSP50 in HCC progression. These results may provide new ideas for the treatment of human HCCs with TSP50 as the target.

\section{Materials and methods \\ UALCAN database and GEPIA database analysis}

The expression level of the TSP50 gene in HCC and normal tissues was analyzed in UALCAN (http://ualcan. path.uab.edu/). Meanwhile, the database was used to analyze the expression of TSP50 in various tumor subgroups based on individual cancer stages, gender, age, and tumor grade. The correlation between TSP50 mRNA expression and survival of HCC patients was analyzed by the GEPIA database (http://gepia.cancer-pku.cn/). GEPIA searches for relationships between gene expression and patient prognosis, such as RFS, across a large collection of publicly available HCC RNA-seq datasets.

\section{Cell culture and plasmid transfection}

L02 cells, Huh7 cells, Bel7402 cells and HEK-293T cells were obtained from the Chinese Academy of Sciences. Both L02 and Bel7402 cell lines used were pure. All cells were authenticated and tested for mycoplasma contamination. L02 cells were cultured in Roswell Park 
Memorial Institute 1640 medium supplemented with 20\% fetal bovine serum (FBS). Huh7 cells and Bel7402 cells were cultured in RPMI 1640 medium with 10\% FBS. The HEK-293T cells were cultured in H-DMEM medium supplemented with $10 \%$ FBS. All mediums were supplemented with 100 units $/ \mathrm{ml}$ penicillin and $100 \mathrm{mg} / \mathrm{ml}$ streptomycin. Cells were maintained at $37^{\circ} \mathrm{C}$ in incubator containing $5 \% \mathrm{CO}_{2}$. When the cells confluence reached $80 \%, 200 \mu \mathrm{l}$ RPMI 1640 serum-free medium was mixed with $5 \mu \mathrm{l}$ of X-tremeGENE HP and $2 \mu \mathrm{g}$ plasmid for transfection. The primers for plasmid construction were shown in Supplementary Table 1.

\section{qRT-PCR detection and Western blot}

Total RNA was isolated using Trizol reagent and cDNAs were synthesized by a RT-PCR Kit and specific primers were designed for qRT-PCR detection (Supplementary Table 2). The detection conditions and procedures were set according to instructions provided in Sybr green kit. The proteins were extracted with RIPA buffer and isolated by SDS-PAGE and transferred to PVDF membrane. After blocking with 5\% skim milk, the membrane was then incubated using the primary antibodies with suggested dilutions. The immunoblotting was detected by ECL luminous fluid.

\section{MTT and BrdU assay}

The cell viability or proliferation was detected using MTT or BrdU ELISA kit. The cells were seeded in 96-well cell plates $\left(2 \times 10^{3}\right.$ cells, $100 \mu \mathrm{L} /$ well $)$, and three duplicate wells were set for each group. $48 \mathrm{~h}$ after transfection, $20 \mu \mathrm{L}(5 \mathrm{mg} / \mathrm{mL})$ of MTT solution was added to each well, and cells were continuously cultured in the incubator for 4-6 h. Finally, labeling was stopped using DMSO and MTT uptake was measured according to the protocol of the manufacturer. For BrdU analysis, the medium was incubated with a $10 \mu \mathrm{M}$ BrdU labeling solution for $6 \mathrm{~h}$. $48 \mathrm{~h}$ after transfection, BrdU uptake was measured according to the manufacturer's protocol.

\section{Analysis of glucose metabolism}

The transfected cells were seeded into 96-well plates, and the medium was replaced with fresh complete medium after $6 \mathrm{~h}$. The medium was collected to measure the concentration of glucose consumption and lactate production after additional $48 \mathrm{~h}$ of incubation. Glucose and lactate levels were measured using glucose assay kit and lactate detection kit respectively according to the manufacturer's instructions. All values were normalized to protein levels.

\section{Glycolysis level detection}

Levels of lactate dehydrogenase (LD) was analyzed by a lactate dehydrogenase assay kit according to the manufacturer's protocol. The cells were treated with $1.5 \mu \mathrm{M}$ oligomycin, luminometer and ATP detection kit were used to detect intracellular ATP levels in cell lysates. Levels of glucose-6-phosphate and 2-phosphoglycerate were analyzed using G6P Assay Kit and 2-PG ELISA kits respectively according to the manufacturer's protocol. All values were normalized to protein levels.

\section{Oxygen consumption rate and extracellular acidification rate detection}

Oxygen consumption rate (OCR) and extracellular acidification rate (ECAR) were detected using the Seahorse XFp Extracellular Flux Analyzer (Seahorse Bioscience, North Billerica, MA). The cells $\left(1 \times 10^{4}\right)$ were seeded into a Seahorse XFp cell culture microplate. For OCR, oligomycin, p-trifluoromethoxy carbonyl cyanide phenylhydrazone (FCCP), and rotenone were added at the indicated time points. When detecting ECAR, glucose, oligomycin, and 2-deoxyglucose (2-DG) were added in sequence at the indicated time points. Finally, data were analyzed using the Seahorse XFp Wave software. Both ECAR and OCR measurements were normalized to protein levels.

\section{LC-MS/MS analysis of interacting proteins}

The total protein of Huh7 cells was separated by polyacrylamide gel electrophoresis (SDS-PAGE) and the protein bands $(50 \mathrm{kDa})$ were excised after Coomassie brilliant blue staining. After decolorization, dehydration, protein reduction, protein alkylation, enzymolysis and extraction, the processed peptides were analyzed using a liquid chromatography masss pectrometry/mass spectrometry (LC-MS/MS) method to obtain the proteins specifically binding to TSP50 in Huh7 cells and characterization of the peptides was carried out by Sangon Biotech.

\section{Co-immunoprecipitation (Co-IP)}

The Pierce Crosslink Immunoprecipitation kit from Thermo Scientific was used for Co-immunoprecipitation experiments detection according to the manufacturer's protocol.

\section{GST pull-down assay}

The recombinant pGEX-4T-1-GST-TSP50 plasmid was transformed into BL21 competent bacteria, and the monoclonal colonies were selected and cultured in shaker at $37^{\circ} \mathrm{C}$ until $\mathrm{OD} 600=0.6 \sim 0.8$. After $6 \mathrm{~h}$ of induction with $1 \mathrm{mM}$ IPTG, the precipitated bacteria were collected after centrifugation. Finally, GST-tag Protein Purification Kit was used to purify GST-TSP50 protein according to the manufacturer's instructions.

The total protein of Huh7 cells or Bel7402 cells was extracted using RIPA lysate. Then, the target protein was mixed with GST pull-down protein binding buffer, well- 
balanced 50\% gel suspension and bait protein rolling at $4{ }^{\circ} \mathrm{C}$ overnight. After centrifugation, the supernatant was removed, and GST pull-down binding buffer was added to resuspend the gel to sufficiently wash off unbound protein. Finally, GST pull-down elution buffer was added to gel and incubated for $10 \mathrm{~min}$. After centrifugation at $4{ }^{\circ} \mathrm{C}$ $1000 \mathrm{~g}$ for $2 \mathrm{~min}$, the supernatant was collected for Western blot detection.

\section{Immunofluorescence detection}

Cells were seeded on sterile coverslips in six-well culture plates. After $24 \mathrm{~h}$, the cells were fixed in $4 \%$ paraformaldehyde and washed three times with PBS. Then, $0.1 \%$ Triton $\mathrm{X}-100$ was used to permeate cells at room temperature for $5 \mathrm{~min}$, and non-specific sites were then blocked with 5\% bovine serum albumin for $30 \mathrm{~min}$. Thereafter, primary antibodies were flooded over the cells, and the culture was incubated at $4{ }^{\circ} \mathrm{C}$ overnight. Furthermore, the cells were incubated with FITC or HRP-conjugated secondary antibody for $1 \mathrm{~h}$ in the dark at room temperature. Nuclei were stained with DAPI for $5 \mathrm{~min}$ at room temperature. Finally, fluorescence analysis was performed using an Olympus BX50 fluorescence microscope (Olympus, Tokyo, Japan).

\section{PKM2 oligomerization assay}

An equal amount of whole cell lysate was collected and cross-linked with $0.025 \%$ glutaraldehyde at $37^{\circ} \mathrm{C}$ for $3 \mathrm{~min}$, and then the reaction was terminated with Tris. $\mathrm{HCl}$ (pH 8.0, $50 \mathrm{mM}$ ). Finally, the processed samples were boiled and analyzed with Western blotting using the indicated antibodies.

\section{PK activity assay}

The cells were collected into the centrifuge tube and the supernatant was discarded after centrifugation. The cells were broken by ultrasound (ice bath, power $20 \%$ or $200 \mathrm{~W}$, ultrasound for $3 \mathrm{~s}, 10 \mathrm{~s}$ intervals, repeated 30 times) for PK extraction. After centrifugation at $8000 \mathrm{~g}$, $4{ }^{\circ} \mathrm{C}$ for $10 \mathrm{~min}$, the supernatant was taken for further detection. The working solution and the cell lysate were mixed and incubated, and the PK activity was measured using a colorimetric-based Pyruvate kinase (PK) activity detection kit according to the manufacturer's protocol.

\section{Lentiviral packaging and tumorigenicity studies}

HEK-293T cells were cultured in H-DMEM medium supplemented with $10 \%$ FBS. Human TSP50, PKM2 or PKM2 K433R gene was cloned into Plvx-AcGFP-N1 to construct vector plasmids. Vector plasmids, packaging genome plasmids pMD2G and pSPAX2 were cotransfected into $293 \mathrm{~T}$ cells. After $48 \mathrm{~h}$, the supernatants of the culture medium were collected and filtered by $0.45 \mu \mathrm{m}$ filter. The virus was concentrated by ultracentrifugation and stored at $-80^{\circ} \mathrm{C}$.
All animal studies were conducted with approval from the Animal Research Ethics Committee of Northeast Normal University (NENU/IACUC, AP20191225) of China and performed in accordance with established guidelines. Female BALB/C nude mice (6 weeks old) were purchased from Charles River Animal Company of China, and kept in a non-pathogenic condition. The mice were randomly blindly assigned to experimental groups $(n=6$ in each group). Lentivirus-infected L02 cells were digested with trypsin after $48 \mathrm{~h}$, washed and resuspended in PBS at $5 \times 10^{7}$ cells $/ \mathrm{ml}$. Then, $200 \mu \mathrm{l}$ of treated cells were injected subcutaneously into female nude mice. After 4 weeks, the mice were sacrificed with cervical dislocation, and the xenografts were resected and weighed. The volume of xenograft was measured with vernier caliper and calculated by the following equation: $\mathrm{V}=\mathrm{L} \times \mathrm{W}^{2} \times$ 0.5 ( $\mathrm{L}$, length; W, width of the xenografts).

\section{Statistical analysis}

IBM SPSS Statistics was used for statistical analysis. The data were presented as mean \pm S.D. All data were from three independent experiments. $P$-values were calculated from Two-sided Student's $t$-test test or one-way ANOVA, ${ }^{*} P<0.05$ and ${ }^{* *} P<0.01$ were displayed as statistical significance, ns, no significant.

\section{Acknowledgements}

We sincerely appreciate all laboratory members.

\section{Author details}

${ }^{1}$ National Engineering Laboratory for Druggable Gene and Protein Screening, Northeast Normal University, Changchun, China. ${ }^{2}$ Research Center of Agriculture and Medicine Gene Engineering of Ministry of Education Northeast Normal University, Changchun, China. ${ }^{3}$ Key Laboratory of Molecular Epigenetics of the Ministry of Education, Northeast Normal University, Changchun, Jilin, China

\section{Author contributions}

Z.S. and Y.B. performed study concept and design; F.G. and X.Z. performed development of methodology and writing, review and revision of the paper: S.W. provided acquisition, analysis and interpretation of data, and statistical analysis; L.Z., Y.S. and G.W. provided technical and material support. All authors read and approved the final paper.

\section{Funding}

This research was supported by grants from the National Natural Science Foundation of China (No.81502284, 81272242 and 81700709), the Research Foundation of Jilin Provincial Science \& Technology Development (Grant No. 20200201135JC, 20180101139JC and 20180520105JH), the Jilin Province Development and Reform Commission (Grant No. 2020C015), the Fundamental Research Funds for the Central Universities (Grant no. 135130006), the Scientific Research Project of Education Department of Jilin Province (Grant No.JJKH20201177KJ).

\section{Ethical approval}

All animal studies were conducted with approval from the Animal Research Ethics Committee of Northeast Normal University (NENU/IACUC, AP20191225) of China and performed in accordance with established guidelines.

Conflict of interest

The authors declare no competing interests. 


\section{Publisher's note}

Springer Nature remains neutral with regard to jurisdictional claims in published maps and institutional affiliations.

Supplementary information The online version contains supplementary material available at https://doi.org/10.1038/s41419-021-03782-w.

Received: 22 December 2020 Revised: 30 April 2021 Accepted: 30 April 2021

Published online: 20 May 2021

\section{References}

1. Cairns, R. A., Harris, I. S. \& Mak, T. W. Regulation of cancer cell metabolism. Nat. Rev. Cancer 11, 85-95 (2011)

2. Vander Heiden, M. G., Cantley, L. C. \& Thompson, C. B. Understanding the Warburg effect: the metabolic requirements of cell proliferation. Science 324 1029-1033 (2009).

3. Oronsky, B. T. et al. Follow the ATP: tumor energy production: a perspective. Anticancer Agents Med. Chem. 14, 1187-1198 (2014).

4. Beyoglu, D. \& Idle, J. R. The metabolomic window into hepatobiliary disease. J. Hepatol. 59, 842-858 (2013).

5. Beyoglu, D. et al. Tissue metabolomics of hepatocellular carcinoma: tumor energy metabolism and the role of transcriptomic classification. Hepatology 58, 229-238 (2013).

6. Kitamura, K. et al. Proliferative activity in hepatocellular carcinoma is closely correlated with glucose metabolism but not angiogenesis. J. Hepatol. 55, 846-857 (2011).

7. Dang, C. V. The interplay between MYC and HIF in the Warburg effect. Ernst Scher. Found. Symp. Proc. 4, 35-53 (2007).

8. Manning, B. D. \& Cantley, L. C. AKT/PKB signaling: navigating downstream. Cell 129, 1261-1274 (2007).

9. Buzzai, M. et al. The glucose dependence of Akt-transformed cells can be reversed by pharmacologic activation of fatty acid beta-oxidation. Oncogene 24, 4165-4173 (2005).

10. Boroughs, L. K. \& DeBerardinis, R. J. Metabolic pathways promoting cancer cell survival and growth. Nat. cell Biol. 17, 351-359 (2015).

11. Koppenol, W. H., Bounds, P. L. \& Dang, C. V. Otto Warburg's contributions to current concepts of cancer metabolism. Nat. Rev. Cancer 11, 325-337 (2011).

12. Zhou, Q. et al. [Gefitinib inhibits glycolysis and induces programmed cell death in non-small cell lung cancer cells]. Nan fang. yi ke da xue xue bao $\mathbf{4 0}$, 884-892 (2020).

13. Gill, K. S. et al. Glycolysis inhibition as a cancer treatment and its role in an antitumour immune response. Biochim. Biophys. Acta 1866, 87-105 (2016).

14. Zhang, D. et al. 2-Deoxy-D-glucose targeting of glucose metabolism in cancer cells as a potential therapy. Cancer Lett. 355, 176-183 (2014).

15. Pelicano, H., Martin, D. S., Xu, R. H. \& Huang, P. Glycolysis inhibition for anticancer treatment. Oncogene 25, 4633-4646 (2006).

16. Song, Z. B. et al. Testes-specific protease 50 promotes cell proliferation via inhibiting activin signaling. Oncogene 36, 5948-5957 (2017).

17. Liu, Y. L. \& Sun, Y. N. Down-regulation of testes-specific protease 50 induces apoptosis in human laryngocarcinoma HEp2 cells in a NF-kappaB-mediated pathway. Mol. Biol. Rep. 41, 7743-7747 (2014).

18. Zheng, L., Xie, G., Duan, G., Yan, X. \& Li, Q. High expression of testes-specific protease 50 is associated with poor prognosis in colorectal carcinoma. PLoS ONE 6, e22203 (2011).

19. Yuan, J. et al. TSP50 depends on its threonine protease activity and its interactions with TNF-alpha-induced NF-kappaB for its role in human cervical tumorigenesis. Cell Biochem. Biophys. 71, 891-896 (2015).

20. Liu, F. et al. Overexpression of Testes-Specific Protease 50 (TSP50) Predicts Poor Prognosis in Patients with Gastric Cancer. Gastroenterol. Res. Pract. 2014, 498246 (2014)

21. Zhou, L. et al. Knockdown of TSP50 inhibits cell proliferation and induces apoptosis in P19 cells. IUBMB Life 62, 825-832 (2010).

22. Mi, X. G. et al. Alantolactone induces cell apoptosis partially through downregulation of testes-specific protease 50 expression. Toxicol. Lett. 224, 349-355 (2014).

23. Song, Z. B. et al. Testes-specific protease 50 promotes cell invasion and metastasis by increasing NF-kappaB-dependent matrix metalloproteinase-9 expression. Cell Death Dis. 6, e1703 (2015).
24. Song, Z. B. et al. Testes-specific protease 50 (TSP50) promotes cell proliferation through the activation of the nuclear factor kappaB (NF-kappaB) signalling pathway. Biochem. J. 436, 457-467 (2011).

25. Li, Y. Y. et al. The threonine protease activity of testes-specific protease 50 (TSP50) is essential for its function in cell proliferation. PLOS ONE 7, e35030 (2012).

26. Song, Z. B. et al. The catalytic triad of testes-specific protease 50 (TSP50) is essential for its function in cell proliferation. Cell. Signal. 26, 2266-2275 (2014).

27. Cortes-Cros, M. et al. M2 isoform of pyruvate kinase is dispensable for tumor maintenance and growth. Proc. Natl Acad. Sci. USA 110, 489-494 (2013).

28. Christofk, H. R. et al. The M2 splice isoform of pyruvate kinase is important for cancer metabolism and tumour growth. Nature 452, 230-233 (2008).

29. Noguchi, T., Inoue, H. \& Tanaka, T. The M1- and M2-type isozymes of rat pyruvate kinase are produced from the same gene by alternative RNA splicing. J. Biol. Chem. 261, 13807-13812 (1986).

30. Luo, W. \& Semenza, G. L. Emerging roles of PKM2 in cell metabolism and cancer progression. Trends Endocrinol. Metab. 23, 560-566 (2012).

31. Zahra, K, Dey, T., Ashish, Mishra, S. P. \& Pandey, U. Pyruvate kinase M2 and cancer: the role of PKM2 in promoting tumorigenesis. Front. Oncol. 10, 159 (2020).

32. Iqbal, M. A., Gupta, V., Gopinath, P., Mazurek, S. \& Bamezai, R. N. Pyruvate kinase M2 and cancer: an updated assessment. FEBS Lett. 588, 2685-2692 (2014).

33. Wong, N., Ojo, D., Yan, J. \& Tang, D. PKM2 contributes to cancer metabolism. Cancer Lett. 356, 184-191 (2015). (2 Pt A).

34. Anastasiou, D. et al. Pyruvate kinase $\mathrm{M} 2$ activators promote tetramer formation and suppress tumorigenesis. Nat. Chem. Biol. 8, 839-847 (2012).

35. Wang, P., Sun, C., Zhu, T. \& Xu, Y. Structural insight into mechanisms for dynamic regulation of PKM2. Protein cell 6, 275-287 (2015).

36. Chandrashekar, D. S. et al. UALCAN: a portal for facilitating tumor subgroup gene expression and survival analyses. Neoplasia 19, 649-658 (2017).

37. Tang, Z. et al. GEPIA: a web server for cancer and normal gene expression profiling and interactive analyses. Nucleic Acids Res. 45, W98-W102 (2017).

38. Lunt, S. Y., Vander \& Heiden, M. G. Aerobic glycolysis: meeting the metabolic requirements of cell proliferation. Annu. Rev. Cell Dev. Biol. 27, 441-464 (2011).

39. Dayton, T. L., Jacks, T. \& Vander Heiden, M. G. PKM2, cancer metabolism, and the road ahead. EMBO Rep. 17, 1721-1730 (2016).

40. Luo, W. et al. Pyruvate kinase M2 is a PHD3-stimulated coactivator for hypoxiainducible factor 1. Cell 145, 732-744 (2011).

41. Yu, Z. et al. Proviral insertion in murine lymphomas 2 (PIM2) oncogene phosphorylates pyruvate kinase M2 (PKM2) and promotes glycolysis in cancer cells. J. Biol. Chem. 288, 35406-35416 (2013).

42. Hitosugi, T. et al. Tyrosine phosphorylation of mitochondrial pyruvate dehydrogenase kinase 1 is important for cancer metabolism. Mol. Cell 44, 864-877 (2011).

43. LV, L. et al. Mitogenic and oncogenic stimulation of K433 acetylation promotes PKM2 protein kinase activity and nuclear localization. Mol. Cell 52, 340-352 (2013).

44. LV, L. et al. Acetylation targets the M2 isoform of pyruvate kinase for degradation through chaperone-mediated autophagy and promotes tumor growth. Mol. Cell 42, 719-730 (2011).

45. Wang, Y. et al. O-GlcNAcylation destabilizes the active tetrameric PKM2 to promote the Warburg effect. Proc. Natl Acad. Sci. USA 114, 13732-13737 (2017).

46. Park, S. H. et al. SIRT2-mediated deacetylation and tetramerization of pyruvate kinase directs glycolysis and tumor growth. Cancer Res. 76, 3802-3812 (2016).

47. Zhang, Z. et al. PKM2, function and expression and regulation. Cell Biosci. 9, 52 (2019).

48. labal, M. A. et al. Missense mutations in pyruvate kinase M2 promote cancer metabolism, oxidative endurance, anchorage independence, and tumor growth in a dominant negative manner. J. Biol. Chem. 289, 8098-8105 (2014).

49. Jin, X. et al. Pyruvate kinase M2 promotes the activation of dendritic cells by enhancing IL-12p35 expression. Cell Rep. 31, 107690 (2020).

50. Li, J. et al. Natural product micheliolide $(M C L)$ irreversibly activates pyruvate kinase M2 and suppresses leukemia. J. medicinal Chem. 61, 4155-4164 (2018).

51. Hanahan, D. \& Weinberg, R. A. Hallmarks of cancer: the next generation. Cell 144, 646-674 (2011).

52. Vaupel, P., Schmidberger, H. \& Mayer, A. The Warburg effect: essential part of metabolic reprogramming and central contributor to cancer progression. Int. J. Radiat. Biol. 95, 912-919 (2019).

53. Chen, Z. et al. Co-expression of PKM2 and TRIM 35 predicts survival and recurrence in hepatocellular carcinoma. Oncotarget 6, 2538-2548 (2015). 
54. Wong, C. C. et al. Switching of pyruvate kinase isoform $L$ to $M 2$ promotes metabolic reprogramming in hepatocarcinogenesis. PLOS ONE 9, e115036 (2014).

55. Rihan, M. et al. Pyruvate Kinase M2: a Metabolic Bug in Re-Wiring the Tumor Microenvironment. Cancer Microenviron. 12, 149-167 (2019).

56. Qiao, W. L. et al. Testes-specific protease 50 as an independent risk factor for poor prognosis in patients with non-small cell lung cancer. Oncol. Lett. 15 8796-8804 (2018).

57. lansante, $\mathrm{V}$. et al. PARP14 promotes the Warburg effect in hepatocellular carcinoma by inhibiting JNK1-dependent PKM2 phosphorylation and activation. Nat. Commun. 6, 7882 (2015).

58. Chen, Z. et al. TRIM35 Interacts with pyruvate kinase isoform M2 to suppress the Warburg effect and tumorigenicity in hepatocellular carcinoma. Oncogene 34, 3946-3956 (2015).

59. Keller, K. E., Tan, I. S. \& Lee, Y. S. SAICAR stimulates pyruvate kinase isoform M2 and promotes cancer cell survival in glucose-limited conditions. Science $\mathbf{3 3 8}$ 1069-1072 (2012).
60. Chaneton, B. et al. Serine is a natural ligand and allosteric activator of pyruvate kinase M2. Nature 491, 458-462 (2012).

61. Gu, Z. et al. NEK2 promotes aerobic glycolysis in multiple myeloma through regulating splicing of pyruvate kinase. J. Hematol. Oncol. 10, 17 (2017)

62. Tang, R. et al. MiR-let-7a inhibits cell proliferation, migration, and invasion by down-regulating PKM2 in gastric cancer. Oncotarget 7, 5972-5984 (2016).

63. Kefas, B. et al. Pyruvate kinase $M 2$ is a target of the tumor-suppressive microRNA-326 and regulates the survival of glioma cells. Neuro-Oncol. 12, 1102-1112 (2010).

64. Xie, X. et al. Pyruvate kinase M2 interacts with DNA damage-binding protein 2 and reduces cell survival upon UV irradiation. Biochemical biophysical Res. Commun. 467, 427-433 (2015).

65. Das Gupta, K. et al. Class lla histone deacetylases drive toll-like receptorinducible glycolysis and macrophage inflammatory responses via pyruvate kinase M2. Cell Rep. 30, 2712-2728 (2020). e2718. 